Article

\title{
Increasing Uniformity of Biosurfactant Production in Starmerella bombicola via the Expression of Chimeric Cytochrome P450s
}

\author{
Robin Geys ${ }^{1,+}$, Marilyn De Graeve ${ }^{1,+}$, Sofie Lodens ${ }^{1}$, Jeroen Van Malderen ${ }^{1}$, \\ Christophe Lemmens ${ }^{1}$, Margaux De Smet ${ }^{1}$, Stein Mincke ${ }^{2}{ }^{\mathbb{D}}$, Inge N. A. Van Bogaert ${ }^{1}{ }^{\mathbb{B}}$, \\ Christian Stevens $^{2} \mathbb{D}$, Sofie L. De Maeseneire ${ }^{1} \mathbb{D}$, Sophie L. K. W. Roelants ${ }^{1, * \mathbb{D}}$ and \\ Wim K. G. Soetaert ${ }^{1}$ (D)
}

1 Centre for Industrial Biotechnology and Biocatalysis (InBio.be), Department of Biotechnology, Faculty of Bioscience Engineering, Ghent University, Coupure Links 653, 9000 Ghent, Belgium; robin.geys@gmail.com (R.G.); marilyn.degraeve@ugent.be (M.D.G.); sofie.lodens@ugent.be (S.L.); vanmalderenjeroen@gmail.com (J.V.M.); lemmens.christophe@outlook.com (C.L.); margaux.de.smet95@gmail.com (M.D.S.); inge.vanbogaert@ugent.be (I.N.A.V.B.); sofie.demaeseneire@ugent.be (S.L.D.M.); wim.soetaert@ugent.be (W.K.G.S.)

2 Sustainable Organic Chemistry and Technology, Department of Green Chemistry and Technology, Faculty of Bioscience Engineering, Ghent University, Coupure Links 653, 9000 Ghent, Belgium; stein.mincke@ugent.be (S.M.); Chris.Stevens@UGent.be (C.S.)

* Correspondence: sophie.roelants@ugent.be; Tel.: +32-926-462-26

+ These authors contributed equally to this work.

Received: 17 August 2018; Accepted: 26 September 2018; Published: 3 October 2018 updates

\begin{abstract}
Sophorolipids are one of the best known microbial biosurfactants and are produced by several yeast species. The best studied producer is Starmerella bombicola, a non-pathogenic yeast associated in nature with bumblebees. Sophorolipids are built up of the rare disaccharide sophorose, which is attached to a fatty acid through a glyosidic bound. Sophorolipids produced by S. bombicola mainly contain oleic acid as the incorporated hydrophobic group. Other chain lengths can, to a certain content, be incorporated by feeding the yeast with substrates of alternative chain lengths. However, the efficiency for such substrates is low as compared to the preferred C18 chain length and defined by the substrate specificity of the first enzymatic step in sophorolipid biosynthesis, i.e., the cytochrome P450 enzyme CYP52M1. To increase product uniformity and diversity at the same time, a new strain of $S$. bombicola was developed that produces sophorolipids with a palmitic acid acyl chain. This was achieved by heterologous expression of the cytochrome P450 cyp1 gene of Ustilago maydis and feeding with palmitic acid. Optimization of the production was done by protein and process engineering.
\end{abstract}

Keywords: sophorolipid; biosurfactant; Starmerella bombicola; cytochrome P450; chimeragenesis; enzyme engineering; process engineering; fermentation; purification; NMR

\section{Introduction}

Starmerella bombicola is a yeast known for its efficient production of sophorolipids, a kind of naturally produced glycolipid biosurfactants [1]. The first step in the sophorolipid biosynthesis involves hydroxylation of a fatty acid by a cytochrome P450 [2]. Cytochrome P450 enzymes are hemeproteins known for their capacity to use molecular oxygen to be incorporated in a wide variety of substrates. In addition to oxygenation reactions, other less common substrates like nitration are also catalyzed by these enzymes [3]. 
In sophorolipid biosynthesis, oxygenation of the fatty acids is carried out by the CYP52M1 enzyme, and it largely determines the length of the fatty acids incorporated into the sophorolipids. The enzyme has a high specificity toward fatty acids like stearic acid (18 carbons, zero double bonds, C18:0) and oleic acid (C18:1), and less toward shorter fatty acids like palmitic (C16:0) or myristic (C14:0) acid. In combination with the specificity of the first uridine diphosphate (UDP) glucosyltransferase (UGTA1), this results in mainly $\mathrm{C} 18: 0$ and $\mathrm{C} 18: 1$, some $\mathrm{C} 18: 2$ and C16:0 fatty acids being incorporated [4]. Therefore, most production processes are conducted with vegetable oils rich in oleic acids, such as rapeseed oil or high-oleic sunflower oil, or with longer fatty acids. Though the incorporation of these longer fatty acids is very efficient, it is also a burden when molecular diversification is desired. Although, it is feasible to incorporate shorter alkyl chains via chemical or biocatalytical methods, in vivo production is still inefficient [5].

Growth trials with shorter fatty acids mainly resulted in long-chain sophorolipids and some smaller amounts of medium-chained ones [6]. This is due to the elongation of the shorter fatty acids by elongase enzymes of the endoplasmic reticulum. Shorter fatty acids can also be degraded in the $\beta$-oxidation pathway and those building blocks will serve as a substrate for de novo synthesis of fatty acids [7]. However, when using specialized substrates like alcohols or ketones, short- and medium-chained sophorolipids can be obtained $[6,8]$. The main problems with this are the high cost of these substrates and the relatively low amounts of novel sophorolipids being produced. Engineering of S. bombicola by knocking-out the $\beta$-oxidation or enzymes involved in long-chain alcohol oxidation already improves the production of these novel new-to-nature molecules using cheaper substrates, but the traditional sophorolipids still remain a part of the mixture since de novo fatty-acid synthesis still occurs with subsequent incorporation of these fatty acids into the sophorolipids $[9,10]$.

To summarize, the major factors hindering a uniform and efficient production of new-to-nature molecules are de novo sophorolipid synthesis enzymes with quite strict substrate specificity, resulting in suboptimal production of these new types. To circumvent this problem, one may try to express a heterologous P450 enzyme with the desired substrate specificities. The CYP1 enzyme from Ustilago maydis takes part in cellobiose lipid synthesis via terminal hydroxylation of palmitic acid (C16:0) [11]. Because the enzyme solely catalyzes terminal hydroxylation, issues associated with the stereochemistry of the hydroxy group are circumvented as well. These features render CYP1 a promising candidate for creating sophorolipids with an altered fatty acid chain in S. bombicola.

\section{Materials and Methods}

\subsection{Strains, Plasmids, and Culture Conditions}

Starmerella bombicola ATCC22214 was the wild-type strain used during the experiments. The derived PT36 strain, a ura3 auxotrophic mutant, was used to engineer all the mutants described in this research [12]. As a hydrophobic substrate, rapeseed oil was used at $37.5 \mathrm{~g} / \mathrm{L}$, or another suitable hydrocarbon like ethyl palmitate, palmitic acid, myristic acid, ethyl myristate, lauric acid, or ethyl myristate at concentrations of $5 \mathrm{~g} / \mathrm{L}$ in shake-flask experiments unless otherwise stated. All the substrates were acquired from Sigma-Aldrich (USA).

Bioreactor experiments were carried out using the Biostat ${ }^{\circledR B}$ 3-L vessels (Sartorius BBI-systems; Germany) with 1.5-L working volume using Norprene ${ }^{\circledR} t u b i n g$ (formulation A-60-G, Saint-Gobain). At inoculation, $1 \mathrm{~L}$ of production medium described by Lang was inoculated with $200 \mathrm{~mL}$ of a late exponential culture [13]. The medium had an initial $\mathrm{pH}$ of 5.8 and was allowed to drop spontaneously to $\mathrm{pH} 3.5$ and was kept at this $\mathrm{pH}$ using a $5 \mathrm{M} \mathrm{NaOH}$ solution. Due to the acidification of the culture broth by the yeast itself, no acid must be added. The reaction vessel was kept at $30^{\circ} \mathrm{C}$ and stirred at $600 \mathrm{rpm}$. Aeration was kept at $1 \mathrm{~L} / \mathrm{min}$ and $2 \mathrm{~mL}$ of ethyl palmitate was added manually every day. Daily sampling of glucose concentrations and optical density was performed as stated in the sampling and analysis section. When the glucose concentration dropped below $30 \mathrm{~g} / \mathrm{L}$, a pre-calculated volume from a $60 \%$ glucose stock solution was added to the fermenter to a final concentration of $100 \mathrm{~g} / \mathrm{L}$. 
Larger fermentations were carried out in a 30-L B bioreactor of Braun Biotech International C20-3 (Germany). Aeration and stirrer rates can be found in the results section. Ethyl palmitate was kept on a heating plate with magnetic stirring at a temperature of $60^{\circ} \mathrm{C}$.

\subsection{Molecular Techniques}

\subsubsection{General Techniques}

High-fidelity PCRs were performed with PrimeSTAR HS DNA Polymerase (Takara, Westburg, Leusden, The Netherlands) when fragments were shorter than 3000 base pairs. Longer fragments were amplified with PrimeSTAR GXL DNA Polymerase (Takara, Westburg, Leusden, The Netherlands). For colony PCR, TAQ-polymerase (New England Biolabs) was used. Genomic DNA was isolated using the Sigma Genomic DNA isolation kit (Sigma-Aldrich, Diegem, Belgium).

Primers, plasmids, and final strains were designed using the Clone Manager Basic Version 9 software suite from Sci-ED Software. Primers were ordered at Integrated DNA Technologies (IDT, Leuven, Belgium) and diluted upon arrival to a $10 \mathrm{mM}$ stock solution. Working solutions were derived from these by a $100 \times$ dilution to $10 \mu \mathrm{mol}$. Sequencing of plasmids and intermediate constructs was performed by LGC Genomics (Berlin, Germany). Used primers are listed in Table 1.

Table 1. Primers used during the research for the creation of the different Starmerella bombicola strains.

\begin{tabular}{cr}
\hline Primer Name & Primer Sequence \\
\hline P52 & ATATGTACTTTTCAATATGATAAACGGAGAAATAACG \\
P69 & GTTTCTTAGCCTCCCATGGAAG \\
P395 & TGAAAAGTACATATGGTACCATGTCGCTCAAAGTGCAG \\
P396 & AACGCTAGCTTGGCGTTATCTAGTGCCTTCCTTGCAAC \\
P722 & AAAATTCCGCTTGGCGGTATTCCTTCACC \\
P723 & GGGAGGCTAAGAAACTTACCCAGCCCACACGTCT \\
P788 & GCCAAGCGGAATTTTCTAGTGCCTTCCTTGCAACATCGAAGGG \\
P847 & CGTTGTCAAGTCCTAAGGTAT \\
P887 & AAGCGTGAAGCTCCTCTGACAATC \\
P1439 & GTTTTCTGCCTTTTTGCGTAC \\
P1641 & CGGAGACGGAAGCCAAATGGCACCTGCCGTTGAAGCAACG \\
P1642 & CAAATACGGAGTAGCGAACGCCTTTTACTTCATCAGCA \\
P1643 & CGTTGCTTCAACGGCAGGTGCCATTTGGCTTCCGTCTCCG \\
P1644 & TGCTGATGAAGTAAAAGGCGTTCGCTACTCCGTATTTG \\
P1645 & GCAAAACGTTTAACAATGCTTGAACTGCTTGAAAAATACC \\
P1646 & CCGGGTATTTTTCAAGCAGTTCAAGCATTGTTAAACGTTTTGCC \\
\hline
\end{tabular}

Transformation of S. bombicola was done following the protocol of Saerens et al. [14]. Used and made strains are listed in Table 2.

Table 2. Strains used and created during the research. NADPH-reduced nicotinamide adenine dinucleotide phosphate; FMN-flavin mononucleotide.

\begin{tabular}{cc}
\hline Strain & Characteristics \\
\hline S. bombicola $\triangle$ cyp $52 m 1$ & Knock-out strain of cyp52m1 [2] \\
S. bombicola cyp1 & Knock-in of cyp1 at the cyp52m1 locus \\
S. bombicola cyp1-bmr & Knock-in of cyp1bmr at the cyp52m1 locus \\
$\begin{array}{c}\text { S. bombicola cyp1-nadph } \\
\text { S. bombicola cyp1-fmn }\end{array}$ & Knock-in of cyp1bmr with a mutation in the NADPH binding domain at the cyp52m1 locus \\
S. bombicola cyp1-double & Knock-in of cyp1bmr with a mutation in the FMN domain at the cyp52m1 locus \\
& Knock-in of cyp1bmr with a mutation in both the NADPH binding domain and the FMN \\
domain at the cyp52m1 locus
\end{tabular}

\subsubsection{Vector Construction}

All the plasmids and derived expression cassettes constructed in this research were targeted toward the cyp $52 m 1$ locus (Figure S1). The P 450 of interest was ligated between the cyp $52 m 1$ promoter 
and terminator. Selection was performed with the ura3 marker which is situated downstream of the P450 coding sequence. Targeting of the expression cassette was performed using homologous regions at the $5^{\prime}$ and $3^{\prime}$ sides of the linear cassette, which had a length of $1000 \mathrm{bp}$.

\subsubsection{Heterologous Expression of Wild-Type Ustilago Maydis CYP1}

The wild-type coding sequence of cyp1 from $U$. maydis 521 was ordered from GeneArt (Figure S2) [11]. No codon optimization was performed, since the codon usage was similar to the codon usage of S. bombicola. The sequence was amplified with primers P395 and P396 (2041 bp). Primers P52 and P69 were used to amplify the cyp $52 m 1$ locus (7514 bp). Ligation was performed by the In-Fusion HD Cloning kit. Transformation of Escherichia coli was performed as described by Sambrook and Russel [15]. This resulted in plasmid BS_EC_0058. After sequencing, the expression cassette was amplified from the plasmid with primers P847 and P947 (6512 bp). After transformation in S. bombicola PT36 and selection on synthetic defined (SD) medium, the transformants were screened for correct integration of the cassette by yeast colony PCR using primers P954 and P679 for the $5^{\prime}$ region and P953 and $\mathrm{P} 95$ for the $3^{\prime}$ region. The $5^{\prime}$ region fragment had a length of $1116 \mathrm{bp}$, and the $3^{\prime}$ region fragment had a length of $1172 \mathrm{bp}$.

\subsubsection{CYP1 Chimeric Construct}

The earlier constructed plasmid BS_EC_0058 was used as a vector backbone to insert the Bacillus megaterium CYP102A1 reductase domain. The vector backbone was amplified with primers P788 and P69 (8558 bp). The reductase of B. megaterium ATCC14581 was amplified from genomic material with primers P722 and P723 (1794 bp) and ligated using the In-Fusion HD Cloning Kit (Takara, Westburg, Leusden, The Netherlands). This resulted in plasmid BS_EC_0091. The expression cassette was amplified with primers P847 and P887 (7310 bp).

\subsubsection{Validation of CYP1BMR Chimeric Construct}

Validation of the function of the reductase domain of B. megaterium ATCC14581 was done by introducing several mutations into the domains of the reductase. Part of the reductase was ordered as a gBlock from IDT (Leuven, Belgium) (Figure S3). For the mutation in the flavin mononucleotide (FMN) domain, primers P1645 and P1642 were used for the vector backbone (9657 bp) with plasmid BS_EC_0091 as a template. The mutated sequence was amplified from the gBlock with primers P1644 and P1646 (761 bp). A similar strategy was followed for the mutation in the reduced nicotinamide adenosine dinucleotide phosphate (NADPH)-binding domain with primers P1642 and P1645 for the vector backbone with BS_EC_0091 (9766 bp) as a template and primers P1643 and P1645 for the mutated sequence $(645 \mathrm{bp})$. Finally, the double-mutation construct was created with primers P1641 and P1642 for linearization of the vector BS_EC_0091 (9043 bp) and primer pair P1643 and P1644 for the sequence carrying the double mutation (1372 bp). All vectors were assembled by Gibson assembly [16].

\subsection{Sampling and Analysis}

\subsubsection{Follow-Up of Growth and Glucose Consumption}

Biomass formation was followed by measuring the optical density at $600 \mathrm{~nm}$ using the FLUOstar OPTIMA fluorometer (BMG LABTECH GmbH, Ortenberg, Germany). Samples were diluted in 0.9\% $\mathrm{NaCl}$ in distilled $\mathrm{H}_{2} \mathrm{O}$ solution if necessary. Follow-up of the glucose concentration was done with the 2700 Select Biochemistry Analyzer (YSI Inc., Yellow Springs, OH, USA). Samples were diluted in a range between 0.1 and $7.5 \mathrm{~g} / \mathrm{L}$ to ensure measurements within the linear range of the equipment.

\subsubsection{Analysis of Sophorolipid Production}

Sophorolipid production from shake-flask experiments was followed by sampling every two days. Three volumes of ethanol were added to the culture broth samples. After shaking vigorously, 
the samples were spun down at $14,000 \times g$ for $5 \mathrm{~min}$. The supernatant was analyzed using HPLC in conjunction with an evaporative light scattering detector (ELSD) and LC-MS [6,14].

Recuperation of the sophorolipids from the fermentation broth was done using one of two techniques depending on the used strain. Isolation from productive strains was done by placing the culture broth overnight at $60{ }^{\circ} \mathrm{C}$. This results in a precipitation of the sophorolipids produced. Recovery of sophorolipids from low-producing strains was done via extraction with ethyl acetate.

Thin-layer chromatography (TLC) was performed to evaluate the purity of the sophorolipid mixture after alkaline hydrolysis on Silica gel $60 \mathrm{~F}_{254}$ TLC plates (VWR). Sophorolipids were separated using a chloroform/methanol/water eluent $(65: 15: 2 \mathrm{v} / \mathrm{v} / \mathrm{v})$ and visualized by heating after dipping in $10 \%(v / v) \mathrm{H}_{2} \mathrm{SO}_{4}[17]$.

\subsubsection{Purification of C16:0 Sophorolipids from Culture Broth}

Total extraction and subsequent purification of sophorolipids from the culture broth was performed by adding ethyl acetate to the broth and collecting the ethyl acetate fraction after phase separation. The ethyl acetate was evaporated and the extracted sophorolipids were dissolved in distilled water. Consequently, a hexane washing step was performed to remove remaining oil and fatty acids. The remaining water fraction was extracted again with ethyl acetate. The hexane washing step and ethyl acetate extraction were repeated until no unwanted molecules were present in the sophorolipid mixture. The sophorolipids were dissolved in distilled water and lyophilized until dry.

Alkaline hydrolysis $\left(\mathrm{pH} 12,50 \%(w / w) \mathrm{NaOH}, 100{ }^{\circ} \mathrm{C}, 1.5 \mathrm{~h}\right)$ was performed under reflux on the purified sophorolipid mixture dissolved in distilled water to reduce the complexity by removing the acetylations and lactonizations. Afterward, the $\mathrm{pH}$ was adjusted to $4(5 \mathrm{M} \mathrm{HCl})$ and the sophorolipids were extracted using one volume of methyl tert-butyl ether (TBME). The TBME was evaporated and the extracted sophorolipids were dissolved in distilled water. The obtained hydrolyzed product was lyophilized again until dry. To check the purity of the sophorolipid mixture, both TLC and LC-MS were performed.

\subsubsection{NMR Analysis}

${ }^{1} \mathrm{H}$ and ${ }^{13} \mathrm{C}$ NMR spectra were analyzed at 400 and $100.6 \mathrm{MHz}$ with a Bruker Avance III device (Bruker Biospin GmbH, Rheinstetten, Germany) using an ${ }^{1} \mathrm{H} / \mathrm{BB}$ z-gradient probe (BBO, $5 \mathrm{~mm}$ ). Firstly, $20 \mathrm{mg}$ of the purified product was dissolved in dimethyl sulfoxide- $\mathrm{d}_{6}\left(\right.$ DMSO- $\left._{6}\right)$, which was also used as an internal chemical shift standard (2.50 ppm for ${ }^{1} \mathrm{H}$ and $39.52 \mathrm{ppm}$ for $\left.{ }^{13} \mathrm{C}\right)$. The spectra $\left({ }^{13} \mathrm{C}, \mathrm{COSY}\right.$, and HSQC) were acquired through the standard sequences available in the Bruker pulse program library and processed using the TOPSPIN 3.2. APT software. Custom settings were used for HMBC (32 scans), TOCSY (100 ms MLEV spinlock, $0.1 \mathrm{~s}$ mixing time, 1.27 s relaxation delay, 16 scans) and H2BC (21.8 ms mixing time, 1.5 s relaxation delay, 16 scans), according to literature $[18,19]$.

\section{Results}

\subsection{Expression of Wild-Type CYP1 from U. maydis in S. bombicola}

The native cyp $52 m 1$ gene of $S$. bombicola was replaced by the cyp 1 gene of $U$. maydis in S. bombicola as described in the Materials and Methods section. To investigate the activity of CYP1 in S. bombicola, an initial growth trial was performed on palmitic acid $(5 \mathrm{~g} / \mathrm{L})$. A strain not capable of synthesizing sophorolipids (a cyp52m1 knock-out, see Table 2) was used as the control. The LC-MS spectrum of samples for the new strain in contrast to the control showed a signal for sophorolipids with molecular mass $679 \mathrm{~m} / z$ (di-acetylated acidic sophorolipids with a C16:0 fatty acid chain; Figure 1). Its formic acid adduct can also be seen $(723 \mathrm{~m} / z)$. This shows that replacement of CYP52M1 by another P450 results in sophorolipid production, with a different alkyl chain in comparison to the wild type. It has to be noted that the amount of produced sophorolipids was very low. After eight days of production, the signal was barely above the detection limit of the LC-MS. 
$\triangle \mathrm{CYP52M1,CYP1}$

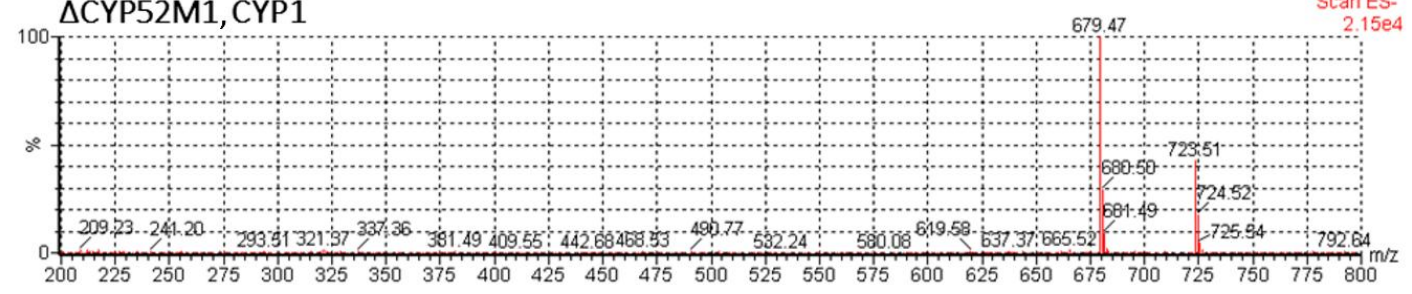

Figure 1. Mass spectrum scan at 24.5 min shows a signal of 16 carbons, zero double bonds (C16:0) di-acetylated acidic sophorolipids $(679 \mathrm{~m} / \mathrm{z})$.

\subsection{Production Analysis of the CYP1 Chimeric P450}

Although proof of concept was, thus, delivered, the production efficiency was very low. This can be due to several effects, e.g., non-efficient electron transfer. CYP1 is a class II P450 requiring assistance of a cytochrome P450 reductase (CPR) for delivery of electrons. Incompatibility of the $\mathrm{CPR}$ can result in low or no electron transfer. Coupling a reductase to the P450 can help achieve electron transfer. Therefore, introduction of a chimeric CYP1 (called CYP1BMR) in S. bombicola was performed. The reductase domain was taken from CYP102A1 from Bacillus megaterium along with the original CYP102A1 linker. The sophorolipid production was investigated on palmitic acid (Figure 2), and indeed, increased amounts of C16:0 sophorolipids were detected for the strain with the CYP1BMR construct compared to the first designed CYP1 strain, but still much lower than regular wild-type sophorolipid production. LC-MS analysis revealed that other congeners were present as well, mostly non- and mono-acetylated variants of the C16:0 sophorolipids.

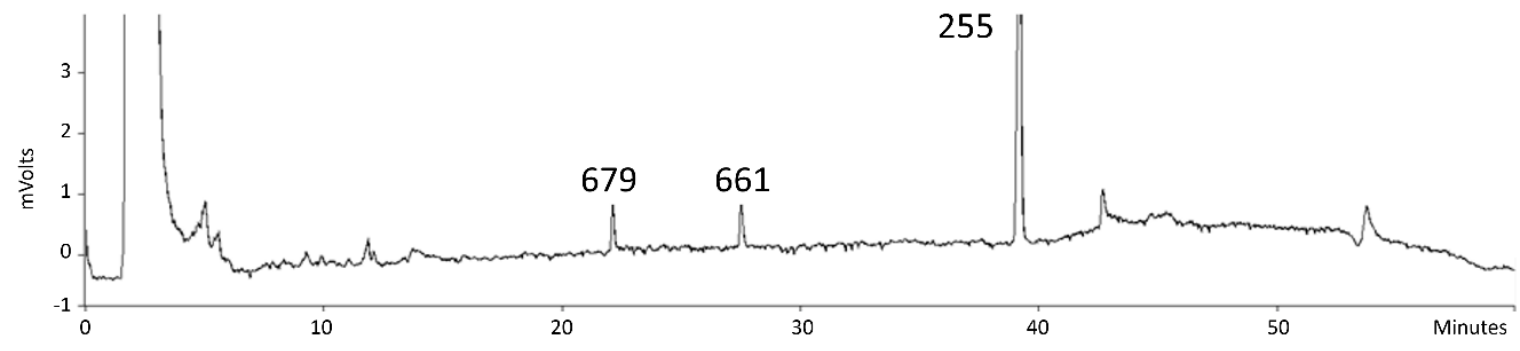

Figure 2. HPLC in conjunction with an evaporative light scattering detector (ELSD) chromatogram of the sophorolipid production of the CYP1BMR strain grown on palmitic acid after eight days in the stationary phase. Products detected are di-acetylated acidic C16:0 sophorolipids $(679 \mathrm{~m} / z, 22.2 \mathrm{~min})$, di-acetylated lactonic C16:0 sophorolipids $(661 \mathrm{~m} / \mathrm{z}, 27.5 \mathrm{~min})$, and the substrate palmitic acid $(255 \mathrm{~m} / \mathrm{z}$, $39.2 \mathrm{~min})$.

\subsection{Validation of the CPR_BMR Functionality}

In order to confirm that the introduction of the CPR was indeed responsible for the increased productivities, several mutants were generated (Table 2). Mutating the flavin adenine dinucleotide (FAD)/FMN-containing cytochrome P450 reductase (BMR) domain at essential residues would render it less efficient in transporting the electrons to the P450, which should result in lower production levels, proving that the BMR domain is responsible for electron donation. Essential residues in the FMN domain are the glycine at position 570, the tryptophan at 574, and the tyrosine at 536 in CYP102A1 [20]. Similar to the FMN domain, several residues exist in the NADPH-binding domain that have an effect on electron transfer and NADPH/NADP+ binding and stability [21,22]. There, a five-fold decrease in the reduction of cytochrome $\mathrm{c}$ was measured with the C999A mutant. Both single mutants for both domains, as well as a double mutant, were constructed and evaluated. The growth trial was conducted using $5 \mathrm{~g} / \mathrm{L}$ ethyl palmitate as a substrate with both single mutants, the double mutant, and the non-mutated CYP1BMR strain. Sophorolipid production is represented in Figure 3. As can be seen, the mutation in the NADPH domain did not result in a drop in sophorolipid production. The mutation 
in the FMN domain resulted in a five-fold drop, while the double mutant had a 16-fold reduction compared to the non-mutated chimeric CYP1BMR. Though it was expected that both single mutations would result in a reduction of production, the results show that this is not the case. Sequence analysis performed after the production trial showed no reversion of the coding sequence of the NADPH mutant (Figure S4). Overall, these data, combined with the enhanced productivity of CYP1BMR compared with the wild-type non-chimeric P450, show that the BMR domain of CYP102A1 is responsible for the donation of electrons to the CYP1 P450 enzyme.

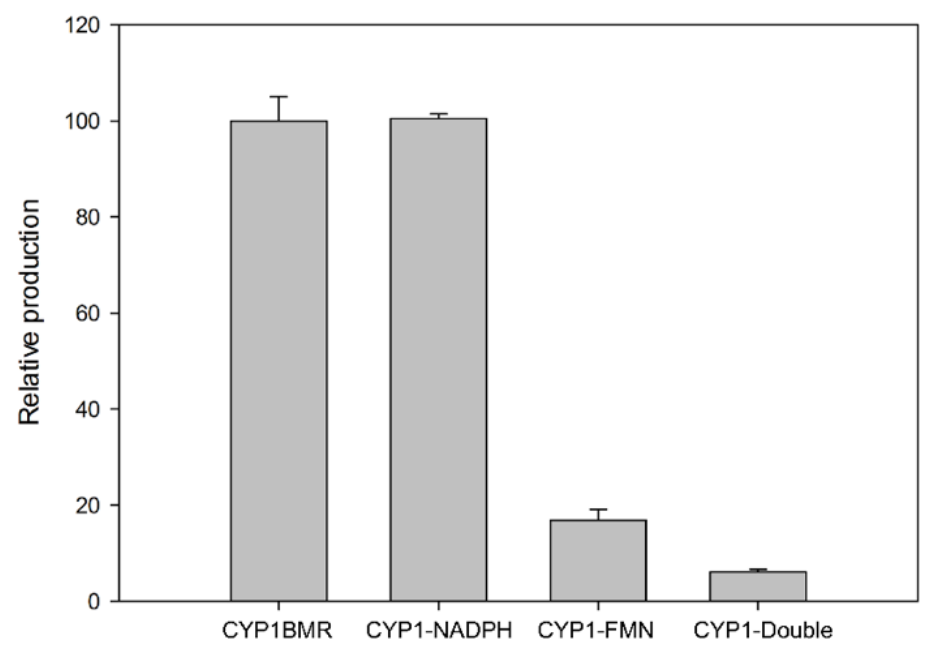

Figure 3. C16:0 sophorolipid production by strains harboring the non-mutated chimeric protein CYP1BMR and mutations in the reduced nicotinamide adenine dinucleotide phosphate (NADPH) binding motif, flavin mononucleotide (FMN) domain, and both domains of the flavin adenine dinucleotide (FAD)/FMN-containing cytochrome P450 reductase (BMR). Error bars represent the standard deviation (SD; $n=3)$.

\subsection{Substrate and Fermentation Optimization of CYP1BMR}

Further optimization of the process was carried out by testing different substrates such as fatty-acid ethyl esters. Substrate accessibility can be a bottleneck during production for some fatty acids as they have high melting temperatures (e.g., palmitic acid: $63^{\circ} \mathrm{C}$ ) and are, thus, solid at $30^{\circ} \mathrm{C}$ (the cultivation temperature). Adding an emulsifier like Tween-20 can improve accessibility, but poses different problems during downstream processing. Ethyl esters of fatty acids are liquid at room temperature and were used in the past for efficient production of sophorolipids [23]. Therefore, a comparison between palmitic acid and ethyl palmitate was conducted and it was found that ethyl palmitate further enhanced productivity at least 50-fold (Figure 4). It has to be noted that the elution times differ from the ones elsewhere due to a shortened HPLC-ELSD analysis protocol to decrease the analysis time. After 11 days in the stationary phase, a final concentration of $1.56 \mathrm{~g} / \mathrm{L}$ was measured. The higher productivity can be explained by the fact that ethyl palmitate is liquid at $30^{\circ} \mathrm{C}$, while palmitic acid is not. The ethyl ester will easily disperse in the broth to form small droplets ensuring a high contact surface. This will result in higher substrate availability for the yeast and enable elevated sophorolipid production. The use of ethyl palmitate did not affect cell viability. During the optimization, several substrate concentrations were used between 5 and $25 \mathrm{~g} / \mathrm{L}$. Increasing the amount of ethyl ester did not further improve production of C16:0 sophorolipids. From the colony-forming units, it became clear that higher amounts of ethyl esters up to $25 \mathrm{~g} / \mathrm{L}$ did not cause a toxic effect which might impair productivity (Figure S5). Therefore, in future shake-flask experiments, a concentration of $5 \mathrm{~g} / \mathrm{L}$ was used. The use of shorter ethyl esters such as ethyl myristate did not result in the production of different sophorolipids than the $\mathrm{C} 16: 0$ ones. 


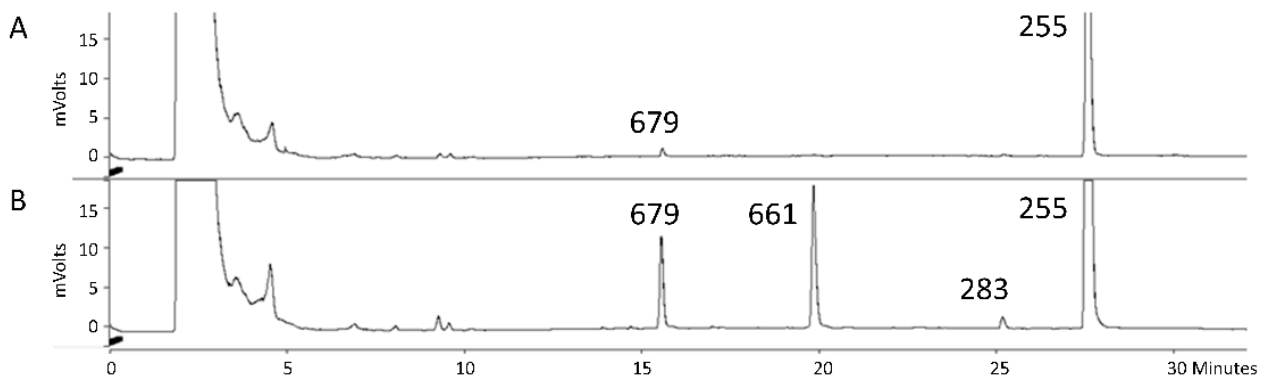

Figure 4. Comparison of the production of C16:0 sophorolipids on palmitic acid (A) versus ethyl palmitate (B). The acidic di-acetylated C16:0 sophorolipids eluted at $15.5 \mathrm{~min}(679 \mathrm{~m} / \mathrm{z})$, and the lactonic di-acetylated C16:0 sophorolipids at $15.8 \mathrm{~min}(661 \mathrm{~m} / \mathrm{z})$. The substrate ethyl palmitate eluted at $25.1 \mathrm{~min}(283 \mathrm{~m} / \mathrm{z})$ while palmitic acid eluted at $27.5 \mathrm{~min}(255 \mathrm{~m} / \mathrm{z})$. It has to be noted that these elution times differ from the ones used elsewhere in this manuscript due to the usage of a shortened HPLC-ELSD analysis protocol.

\subsection{Scale-Up of the CYP1BMR Chimeric Strain}

Scale-up of production of C16:0 sophorolipids was first done in a 3-L and later in a 30-L bioreactor. During this fermentation, heavy foaming occurred, which was problematic. It was observed that this foaming was reduced each time ethyl palmitate was added to the fermenter. Therefore, the addition of hydrophobic substrate was split up into two daily portions of $1 \mathrm{~mL}$. In Figure 5, glucose consumption and average optical density are depicted.

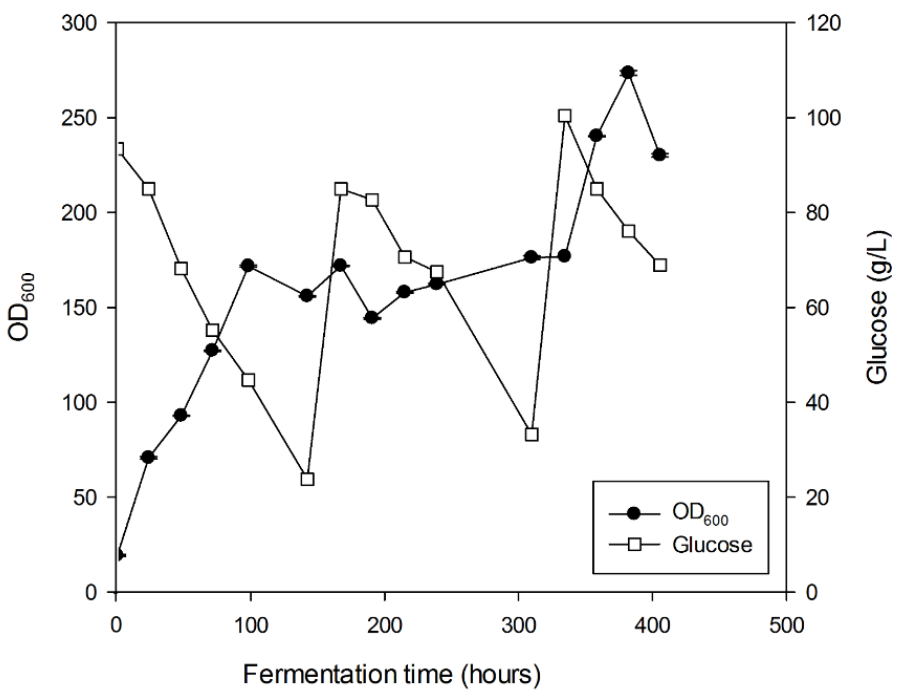

Figure 5. Glucose consumption and optical density for the 3-L fermentation with Starmerella bombicola CYP1BMR using ethyl palmitate.

Depending on the growth phase, glucose consumption rates of $0.5 \mathrm{~g} / \mathrm{L} \cdot \mathrm{h}$ and $0.4 \mathrm{~g} / \mathrm{L} \cdot \mathrm{h}$ were calculated for the exponential and stationary phases, respectively. The entire fermentation lasted 411.4 $\mathrm{h}$ with a total titer of $9.9 \pm 0.1(\mathrm{SD} ; n=3) \mathrm{g} / \mathrm{L}$ of $\mathrm{C} 16: 0$ sophorolipids. This corresponds to a productivity of $24 \pm 0.2$ (SD; $n=3$ ) $\mathrm{mg} / \mathrm{L} \cdot \mathrm{h}$. Analysis by HPLC and LC-MS showed that the major products being produced were the lactonic di-acetylated C16:0 sophorolipids (Figure 6). 


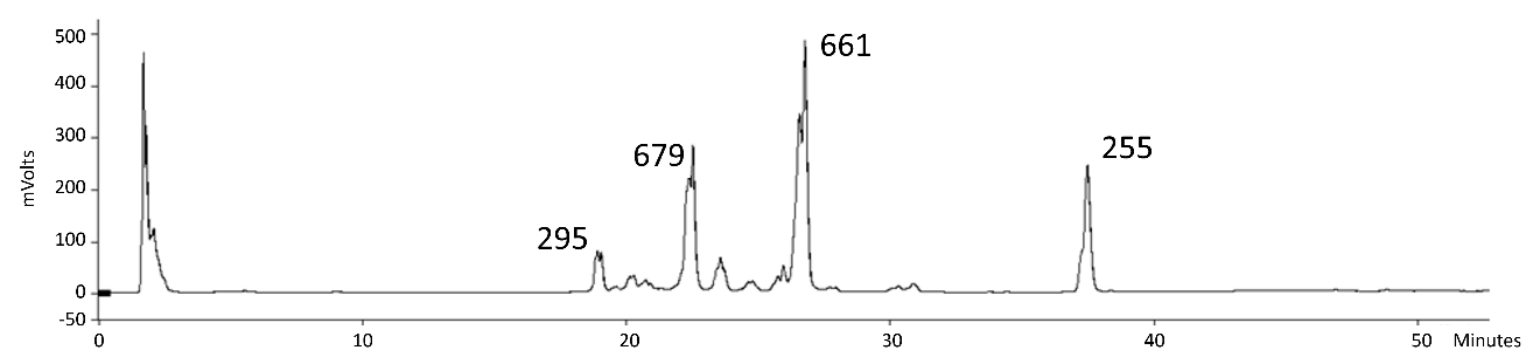

Figure 6. Sophorolipids produced in a 3-L fermenter using ethyl palmitate. At $18.9 \mathrm{~min}$, non-acetylated acidic C16:0 sophorolipids eluted $(295 \mathrm{~m} / z)$. Di-acetylated congeners were present around 22.4 and $26.6 \mathrm{~min}$, corresponding to acidic and lactonic sophorolipids, respectively $(679$ and $661 \mathrm{~m} / \mathrm{z}$ ). Palmitic acid eluted at $37.4(255 \mathrm{~m} / \mathrm{z})$.

Foam formation became even more troublesome on a 30-L scale compared to the 3-L scale. A first 30-L fermentation (fermentation A) was run using the same feeding and stirring strategy as the 3-L fermenter. However, due to the excessive foam formation, aeration had to be reduced to approximately $0.1 \mathrm{vvm}$.

A second 30-L fermentation (fermentation B) used a different feeding strategy. Conventional antifoam methods, such as the use of a silicon-based antifoam, were not desired due to separation problems during downstream processing of these molecules from the sophorolipids. Because, when a small amount of ethyl palmitate was added to the reactor, foam disappeared for $1 \mathrm{~h}$ after which it then slowly started to accumulate again, the substrate was used as an antifoam by adding it every $20 \mathrm{~min}$ in short bursts of $2 \mathrm{~s}$ (equaling $200 \mu \mathrm{L}$ of ethyl palmitate). This way, it gave rise to a relatively foam-free fermentation process. This allowed an overall higher aeration rate in fermentation B compared to A. The parameters can be found in Table 3. As can be seen, due to the new feeding and antifoam strategy, aeration could be increased up to $0.3 \mathrm{vvm}$ in fermentation $\mathrm{B}$.

Table 3. Parameters for 30-L fermentations A and B. The average optical density was calculated for the stationary phase.

\begin{tabular}{ccc}
\hline & Fermentation A (Manual Feed) & Fermentation B (Automatic Feed) \\
\hline Aeration & $\pm 1.2 \mathrm{~L} / \mathrm{min}($ approx. 0.1 vvm) & $\pm 5 \mathrm{~L} / \mathrm{min}($ approx. 0.3 vvm) \\
Stirring rate & $600 \mathrm{rpm}$ & $600 \mathrm{rpm}$ \\
Total glucose consumption & $3492 \mathrm{~g}$ & $2577 \mathrm{~g}$ \\
Total ethyl palmitate & $54.85 \mathrm{~g}$ & $162 \mathrm{~g}$ \\
Duration & $448 \mathrm{~h}$ & $359 \mathrm{~h}$ \\
Average optical density & $131.9 \pm 16.7$ & $120.9 \pm 13.6$ \\
Sophorolipid titers & $9.0 \pm 0.2 \mathrm{~g}$ & $12.1 \pm 0.2 \mathrm{~g}$ \\
Productivity & $20 \pm 0.4 \mathrm{mg} / \mathrm{L} \cdot \mathrm{h}$ & $33 \pm 0.5 \mathrm{mg} / \mathrm{L} \cdot \mathrm{h}$ \\
\hline
\end{tabular}

At the end of both 30-L fermentations, the broth was harvested and stored at $4{ }^{\circ} \mathrm{C}$. After $48 \mathrm{~h}$, a brown precipitation could be observed. HPLC analysis revealed that these were $\mathrm{C} 16: 0$ sophorolipids. When comparing the two fermentations, the ratio of acidic versus lactonic $\mathrm{C} 16: 0$ sophorolipids differed (Figure 7). Fermentation A showed a lower amount of lactonic sophorolipids compared to fermentation B. In literature, aeration was described to affect the saturation degree of the fatty acids incorporated and the lactonization degree of the produced sophorolipids [24-27]. 


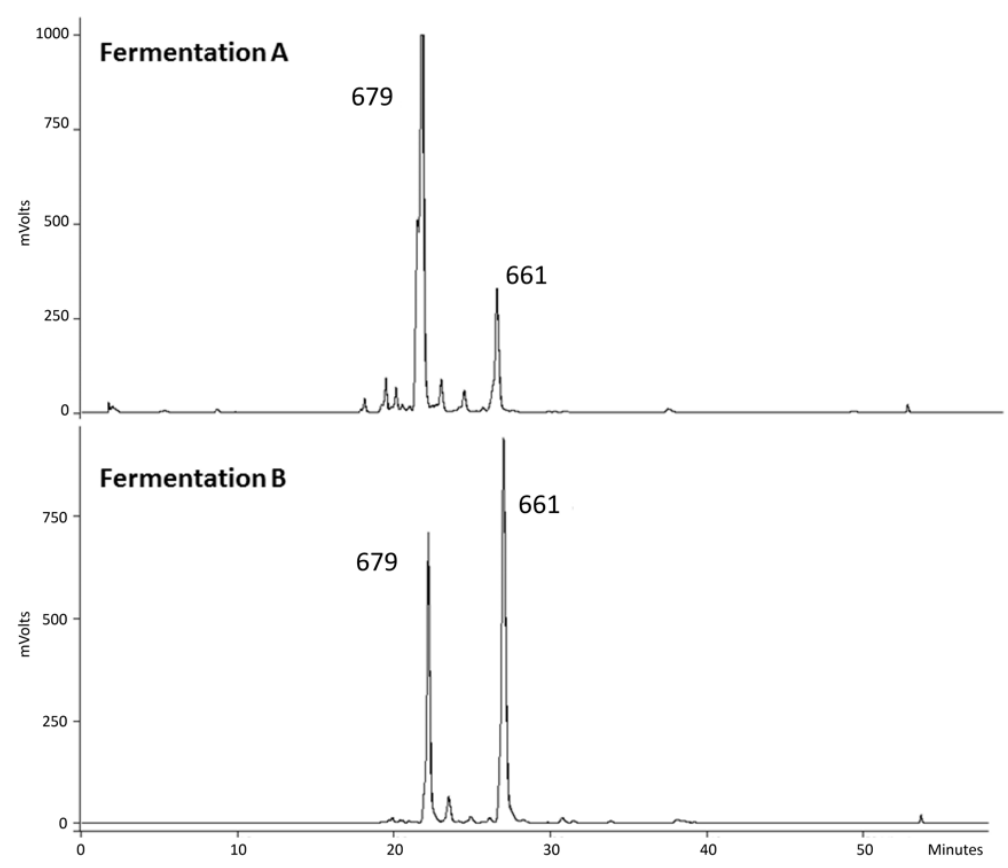

Figure 7. Difference in the ratio of the sophorolipid products in the two 30-L fermentations: acidic (22.4 $\mathrm{min}, 679 \mathrm{~m} / \mathrm{z}$ ) and lactonic $(26.6 \mathrm{~min}, 661 \mathrm{~m} / \mathrm{z}$ ) di-acetylated C16:0 sophorolipids.

\subsection{NMR Structure Elucidation}

Final confirmation of the molecular structure of the produced sophorolipids was done by performing nuclear magnetic resonance spectroscopy (NMR) on the hydrolyzed C16:0 sophorolipids obtained by stepwise purification and alkaline hydrolysis (see Section 2.3.3). This way, a uniform product was obtained with only non-acetylated acidic $\mathrm{C} 16: 0$ sophorolipids.

The chemical formula $\mathrm{C}_{28} \mathrm{H}_{52} \mathrm{O}_{13}$ was confirmed by the integration of the ${ }^{1} \mathrm{H}$ and ${ }^{13} \mathrm{C}$ NMR spectra (Figure S6). When both subterminal and terminal hydroxylation occur, a doublet around $1.12 \mathrm{ppm}$ is detected in the ${ }^{1} \mathrm{H}$ spectrum [28]. Considering that there is no signal that accounts for the presence of a methyl group, here, only terminal hydroxylation occurred. NMR spectral analysis could also validate the presence of two carbohydrates (two anomeric protons) and a terminal carboxyl group (174.66 ppm in the ${ }^{13} \mathrm{C}$ spectrum). The NMR data support the LC-MS identification, and confirm the molecular structure of the "novel" sophorolipids with a terminally bound palmitic acid tail, as shown in Figure 8.

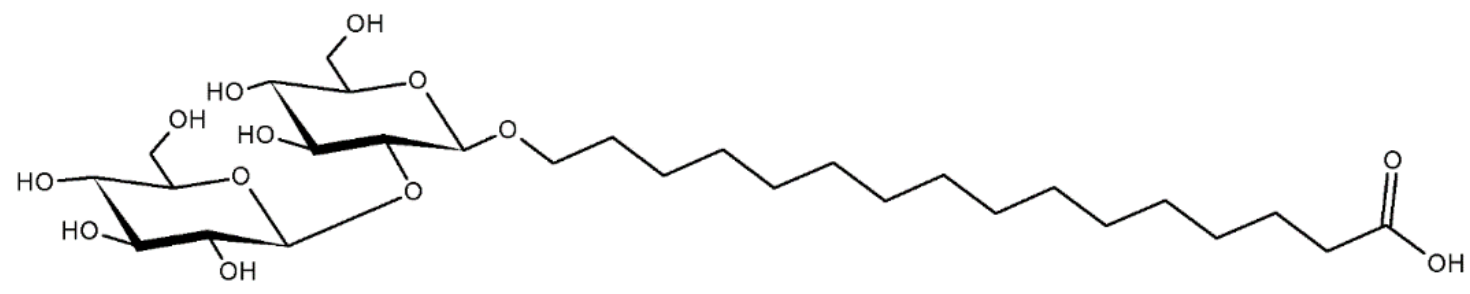

Figure 8. Molecular structure of the novel C16:0 sophorolipids after alkaline hydrolysis.

\section{Discussion}

Introducing a novel cytochrome P450 in S. bombicola to alter the fatty-acid tail of the produced molecules is a valuable tool for creating tailor-made glycolipids. In this research, a shift toward shorter chains of 16 carbon atoms was attained. In the case of CYP1 from $U$. maydis, a low amount of activity (i.e., sophorolipid production) was obtained by heterologous expression of the wild-type CYP1 U. maydis enzyme. To increase production, a strategy to ensure sufficient electron transfer from the CPR to the P450 was evaluated via the generation of a chimeric P450 CYP1 from $U$. maydis with 
the BMR reductase domain of CYP102A1 from B. megaterium. Higher activity was detected for the strain containing the fusion protein, which was confirmed to be caused by the attached BMR domain. Interestingly, for the mutated NADPH-binding domain of BMR, a reduction was only observed when it was combined with a mutation in the FMN domain. Though the exact reason remains unknown, two mutations in the reductase domain might inflict substantial conformational changes, severely impairing its function. Further optimization of the production strain and scale-up of the production process resulted in more efficient production of C16:0 sophorolipids and the generation of $30 \mathrm{~g}$ of C16:0 terminal hydroxylated sophorolipids. The product is currently being used for characterization studies and application research. The overall productivity is still at least 40 -fold lower than several other reported strains [28,29], which shows that additional engineering and optimization is required.

Supplementary Materials: The following are available online at http:/ / www.mdpi.com/2504-5377/2/4/42/s1: Figure S1: Overview of the knock-in strategy at the cyp52m1 locus; Figure S2: Ordered coding sequence of the Ustilago maydis cyp1 gene; Figure S3: Ordered mutated Bacillus megaterium cyp102a1 reductase sequence (BMR); Figure S4: Sequencing of the BMR mutant strains; Figure S5: Effect of concentration of ethyl palmitate on colony-forming units; Figure S6: ${ }^{1} \mathrm{H}$ and ${ }^{13} \mathrm{C}$ NMR spectra of C16:0 terminal hydroxylated sophorolipids.

Author Contributions: This manuscript was generated with an equal contribution from the first and second authors (R.G. and M.D.G.). Conceptualization, R.G., I.N.A.V.B., and W.K.G.S. Methodology, R.G., M.D.G., J.V.M., C.L., M.D.S., and S.M. Validation, R.G., M.D.G., S.L., I.N.A.V.B., S.L.D.M., and S.L.K.W.R. Formal analysis, R.G., M.D.G., J.V.M., C.L., M.D.S., and S.M. Investigation, R.G., M.D.G., J.V.M., C.L., M.D.S., and S.M. Resources, C.S. and W.K.G.S. Writing-original draft preparation, R.G., M.D.G., and S.L. Writing-review and editing, M.D.G., S.L., S.L.D.M., S.L.K.W.R., and W.K.G.S. Visualization, R.G. and M.D.G. Supervision, I.N.A.V.B., S.L.D.M., and S.L.K.W.R.

Funding: This research was funded by the Flemish Agency Flanders Innovation and Entrepreneurship (formerly known as the Agency for Innovation by Science and Technology; Grant No. 121129), the European FP7 project Biosurfing (Grant No. 289219), and the Bio Based Industries Joint Undertaking under the European Union's Horizon 2020 research and innovation program (Grant No. 669003).

Conflicts of Interest: The authors declare no conflicts of interest.

\section{References}

1. Davila, A.-M.M.; Marchal, R.; Vandecasteele, J.-P.P. Sophorose lipid fermentation with differentiated substrate supply for growth and production phases. Appl. Microbiol. Biotechnol. 1997, 47, 496-501. [CrossRef]

2. Van Bogaert, I.N.A.; Holvoet, K.; Roelants, S.L.K.W.; Li, B.; Lin, Y.-C.C.; Van de Peer, Y.; Soetaert, W. The biosynthetic gene cluster for sophorolipids: A biotechnological interesting biosurfactant produced by Starmerella bombicola. Mol. Microbiol. 2013, 88, 501-509. [CrossRef] [PubMed]

3. Barry, S.M.; Kers, J.A.; Johnson, E.G.; Song, L.; Aston, P.R.; Patel, B.; Krasnoff, S.B.; Crane, B.R.; Gibson, D.M.; Loria, R.; et al. Cytochrome P450-catalyzed L-tryptophan nitration in thaxtomin phytotoxin biosynthesis. Nat. Chem. Biol. 2012, 8, 814-816. [CrossRef] [PubMed]

4. Saerens, K.M.J.; Van Bogaert, I.N.A.; Soetaert, W. Characterization of sophorolipid biosynthetic enzymes from Starmerella bombicola. FEMS Yeast Res. 2015, 15, 1-9. [CrossRef] [PubMed]

5. Delbeke, E.I.P.; Movsisyan, M.; Van Geem, K.M.; Stevens, C.V. Chemical and enzymatic modification of sophorolipids. Green Chem. 2016, 18, 76-104. [CrossRef]

6. Van Bogaert, I.; Fleurackers, S.; Van Kerrebroeck, S.; Develter, D.; Soetaert, W. Production of new-to-nature sophorolipids by cultivating the yeast Candida bombicola on unconventional hydrophobic substrates. Biotechnol. Bioeng. 2011, 108, 734-741. [CrossRef] [PubMed]

7. Tehlivets, O.; Scheuringer, K.; Kohlwein, S.D. Fatty acid synthesis and elongation in yeast. Biochim. Biophys. Acta-Mol. Cell Biol. Lipids 2007, 1771, 255-270. [CrossRef] [PubMed]

8. Brakemeier, A.; Wullbrandt, D.; Lang, S. Microbial alkyl-sophorosides based on 1-dodecanol or 2-, 3- or 4-dodecanones. Biotechnol. Lett. 1998, 20, 215-218. [CrossRef] 
9. Van Bogaert, I.N.A.; Sabirova, J.; Develter, D.; Soetaert, W.; Vandamme, E.J. Knocking out the MFE-2 gene of Candida bombicola leads to improved medium-chain sophorolipid production. FEMS Yeast Res. 2009, 9, 610-617. [CrossRef] [PubMed]

10. Takahashi, F.; Igarashi, K.; Hagihara, H. Identification of the fatty alcohol oxidase FAO1 from Starmerella bombicola and improved novel glycolipids production in an FAO1 knockout mutant. Appl. Microbiol. Biotechnol. 2016, 100, 9519-9528. [CrossRef] [PubMed]

11. Teichmann, B.; Linne, U.; Hewald, S.; Marahiel, M.A.; Bölker, M. A biosynthetic gene cluster for a secreted cellobiose lipid with antifungal activity from Ustilago maydis. Mol. Microbiol. 2007, 66, 525-533. [CrossRef] [PubMed]

12. Lodens, S.; De Graeve, M.; Roelants, S.L.K.W.K.W.; De Maeseneire, S.L.; Soetaert, W. Transformation of an exotic yeast species into a platform organism: A case study for engineering glycolipid production in the yeast Starmerella bombicola. In Synthetic Biology: Methods and Protocols; Braham, J.C., Ed.; Springer Humana Press: New York, NY, USA, 2018; pp. 95-123. ISBN 9781493977949.

13. Lang, S.; Brakemeier, A.; Heckmann, R.; Spöckner, S.; Rau, U. Production of native and modified sophorose lipids. Chim Oggi 2000, 18, 76-79.

14. Saerens, K.M.J.; Saey, L.; Soetaert, W. One-Step Production of Unacetylated Sophorolipids by an Acetyltransferase Negative Candida bombicola. Biotechnol. Bioeng. 2011, 108, 2923-2931. [CrossRef] [PubMed]

15. Sambrook, J.; Russell, D. Molecular Cloning: A Laboratory Manual, 3rd ed.; Cold Spring Harbour Laboratory Press: Cold Spring Harbor, NY, USA, 2001.

16. Gibson, D.G.; Young, L.; Chuang, R.-Y.; Venter, J.C.; Hutchison, C.A.; Smith, H.O.; Hutchison, C.A., III.; America, N. Enzymatic assembly of DNA molecules up to several hundred kilobases. Nat. Methods 2009, 6, 343-345. [CrossRef] [PubMed]

17. Asmer, H.-J.; Lang, S.; Wagner, F.; Wray, V. Microbial production, structure elucidation and bioconversion of sophorose lipids. J. Am. Oil Chem. Soc. 1988, 65, 1460-1466. [CrossRef]

18. Petersen, B.O.; Vinogradov, E.; Kay, W.; Würtz, P.; Nyberg, N.T.; Duus, J.; Sørensen, O.W. H2BC: A new technique for NMR analysis of complex carbohydrates. Carbohydr. Res. 2006, 341, 550-556. [CrossRef] [PubMed]

19. Gheysen, K.; Mihai, C.; Conrath, K.; Martins, J.C. Rapid identification of common hexapyranose monosaccharide units by a simple TOCSY matching approach. Chem. A Eur. J. 2008, 14, 8869-8878. [CrossRef] [PubMed]

20. Kleine, M.L.; Fulco, A.J. Critical residues involved in FMN binding and catalytic activity in cytochrome P450BM-3. J. Biol. Chem. 1993, 268, 7553-7561.

21. Cirino, P.C.C.; Arnold, F.H. Regioselectivity and Activity of Cytochrome P450 BM-3 and Mutant F87A in Reactions Driven by Hydrogen Peroxide. Adv. Synth. Catal. 2002, 344, 932-937. [CrossRef]

22. Dietrich, M.; Do, T.A.; Schmid, R.D.; Pleiss, J.; Urlacher, V.B. Altering the regioselectivity of the subterminal fatty acid hydroxylase P450 BM-3 towards gamma- and delta-positions. J. Biotechnol. 2009, 139, 115-117. [CrossRef] [PubMed]

23. Davila, A.-M.; Marchal, R.; Vandecasteele, J.-P. Kinetics and balance of a fermentation free from product inhibition: Sophorose lipid production by Candida bombicola. Appl. Microbiol. Biotechnol. 1992, 38, 6-11. [CrossRef]

24. Van Bogaert, I.N.A.; Saerens, K.; De Muynck, C.; Develter, D.; Soetaert, W.; Vandamme, E.J.; Van Bogaert, I.N.A.; Saerens, K.; De Muynck, C.; Develter, D.; et al. Microbial production and application of sophorolipids. Appl. Microbiol. Biotechnol. 2007, 76, 23-34. [CrossRef] [PubMed]

25. Oliveira, M.R.; Camilios-neto, D.; Baldo, C.; Magri, A.; Celligoi, M.A.P.C. Biosynthesis and Production of Sophorolipids. Int. J. Sci. Technol. Res. 2014, 3, 133-143.

26. Ratsep, P.; Shah, V. Identification and quantification of sophorolipid analogs using ultra-fast liquid chromatography-mass spectrometry. J. Microbiol. Methods 2009, 78, 354-356. [CrossRef] [PubMed]

27. Guilmanov, V.; Ballistreri, A.; Impallomeni, G.; Gross, R.A.; Catania, Á.; Doria, V.A.; Chimiche, S. Oxygen transfer rate and sophorose lipid production by Candida bombicola. Biotechnol. Bioeng. 2002, 77, 489-494. [CrossRef] [PubMed] 
28. Van Renterghem, L.; Roelants, S.L.K.W.; Baccile, N.; Uytersprot, K.; Taelman, M.C.; Everaert, B.; Mincke, S.; Ledegen, S.; Debrouwer, S.; Scholtens, K.; et al. From lab to market: An integrated bioprocess design approach for new-to-nature biosurfactants produced by Starmerella bombicola. Biotechnol. Bioeng. 2018, 115, 1195-1206. [CrossRef] [PubMed]

29. Roelants, S.L.K.W.; Ciesielska, K.; De Maeseneire, S.L.; Moens, H.; Everaert, B.; Verweire, S.; Denon, Q.; Vanlerberghe, B.; Van Bogaert, I.N.A.; Van der Meeren, P.; et al. Towards the industrialization of new biosurfactants: Biotechnological opportunities for the lactone esterase gene from Starmerella bombicola. Biotechnol. Bioeng. 2016, 113, 550-559. [CrossRef] [PubMed]

(C) 2018 by the authors. Licensee MDPI, Basel, Switzerland. This article is an open access article distributed under the terms and conditions of the Creative Commons Attribution (CC BY) license (http:/ / creativecommons.org/licenses/by/4.0/). 